\title{
Analyses of Adverse Drug Reactions Nationwide Active Surveillance Network: Canadian Pharmacogenomics Network for Drug Safety (CPNDS) database
}

\author{
Reo Tanoshima ${ }^{1,2}$, Amna Khan ${ }^{1,2}$, Agnieszka K Biala ${ }^{1,2}$, Jessica N Trueman ${ }^{1,2}$, Britt I Drogemoller ${ }^{3,4}$, \\ Galen Eb Wright ${ }^{2,4}$, Gabriella Ss Groeneweg ${ }^{1,2}$, Colin Jd Ross ${ }^{3}$, Bruce C Carleton ${ }^{1,2}$ \\ ${ }^{I}$ Division of Translational Therapeutics, Department of Pediatrics, Faculty of Medicine, The University of British \\ Columbia, Vancouver, British Columbia, Canada, ${ }^{2}$ BC Children's Hospital Research Institute, Vancouver, British \\ Columbia, Canada, ${ }^{3}$ Faculty of Pharmaceutical Sciences, The University of British Columbia, Vancouver, British \\ Columbia, Canada, ${ }^{4}$ Department of Medical Genetics, Centre for Molecular Medicine and Therapeutics, Faculty of \\ Medicine, The University of British Columbia, Vancouver, British Columbia, Canada
}

\section{Background}

Adverse drug reactions (ADRs) are a major problem in modern medicine, representing the 4th highest cause of mortality in Western society. Pharmacogenomic tests are one of the most effective and promising methods to solve the problems related to ADRs. The pan-Canadian active surveillance network, Canadian Pharmacogenomics Network for Drug Safety (CPNDS), has successfully identified predictive genomic biomarkers of ADRs, especially in the pediatric oncology field. CPNDS is composed of 26 sites across Canada, and clinical information of ADRs is entered into our host database. Patients' biological samples are also collected for genetic analyses.

Objectives

The objective of this study is to describe an overview of the drugs, ADRs, and clinical information housed in the current CPNDS database.

Methods

The clinical information entered into the database between May 15, 2005 and May 9, 2017 was collected and analyzed using Excel $^{\circledR}$ (Microsoft) and SPSS ${ }^{\circledR}$ (IBM). This included demographic information, number of ADR reports, reports of drug use without ADRs (matched controls), date of ADR occurance, suspected drugs, and fatal cases. The reactions were categorized into 38 common and/or clinically important reaction categories.

Results

The CPNDS database consisted of 93,974 reports of medication use. It comprised of 10,475 ADR reports, of which 72.6\% occurred in pediatric patients $(\leq 21 \mathrm{y} / \mathrm{o})$ and $27.4 \%$ were experienced by adults $(>21 \mathrm{y} / 0)$, and 83,499 matched controls. The self-reported ancestry of captured patients were largely from Europe (38.15\%), Canada (9.63\%) and East Asia $(4.86 \%)$. The five most frequent ADRs were skin rash (12.25\%), peripheral neuropathy (11.57\%), cardiotoxicity $(7.16 \%)$, central nervous system toxicity $(6.38 \%)$, ototoxicity $(5.36 \%)$ and the five most common suspected drugs were methotrexate $(9.66 \%)$, vincristine $(7.64 \%)$, doxorubicin (6.84\%), cisplatin (5.00\%), and L-asparaginase (4.64\%). Highly significant and clinically relevant biomarkers of ADRs including anthracycline-induced cardiotoxicity and cisplatininduced hearing loss have been discovered and replicated using the CPNDS database thus far.

Conclusions

Potent pharmacological agents such as cancer chemotherapy used at high-dose to prolong life are dominant ADRs in the database. The CPNDS database is a valuable global resource to identify clinical and genomic predictors of ADRs.

(This work was done by CPNDS consortium.) 